\title{
Lesiones por minas antipersona y munición sin explotar, Hospital Universitario de Neiva, 2005-2009
}

\author{
Injuries caused by anti-personal landmines and unexploded munitions, \\ Hospital Universitario de Neiva 2005-2009
}

\author{
Gilberto Mauricio Astaiza Arias?', Viviana Andrea Calderón Ramírez²
}

\section{Resumen}

Colombia ha vivido un prolongado conflicto armado interno durante a proximadamente cinco décadas. En este, las acciones bélicas se han caracterizado por la alta degradación en las formas de actuar de los diferentes actores armados, causando gran impacto en la morbilidad y mortalidad de la población civil y combatientes.

Con el fin de establecer las características de las lesiones en personas afectadas por las minas antipersonal (MAP) y municiones sin explotar (MUSE) que fueron atendidas quirúrgicamente en el Hospital Universitario de Neiva, entre los años 2005-2009, se efectuó un estudio cuantitativo, descriptivo, serie de casos retrospectivo. Fueron 41 lesionados por las MAP-MUSE atendidos en el Hospital Universitario de Neiva, obtenidos mediante la revisión de los registros de patología y de las historias clínicas. Entre los resultados se encuentra que la mayoría de los afectados por MAP-MUSE son hombres jóvenes militares, cuyos miembros inferiores son los más afectados.

A todos los lesionados se les hizo amputación quirúrgica de la extremidad afectada, y más de la mitad de ellos iniciaron rehabilitación con la adaptación de una prótesis. Este problema de salud pública evidencia la degradación del conflicto armado, con gran impacto en la población de las zonas rurales y amerita profundizar en el cumplimiento de los tratados para la prohibición de estas armas, además de enfatizar en procesos de prevención y de educación en las zonas de riesgo, mientras se busca la solución negociada del conflicto armado interno.

Palabras clave: Minas terrestres, municiones sin explotar, amputación traumática, manejo de trauma, lesiones de miembros inferiores.

\begin{abstract}
Colombia has experienced a prolonged internal armed conflict for about five decades. The military actions have been characterized by high degradation in the way of acting of the different armed groups, causing great impact on morbidity and mortality of the civilian population and combatants.

A quantitative, descriptive, retrospective case series study was conducted in order to establish the characteristics of injuries in people affected by anti-personall and mines (APLM) and unexploded munitions (UXM) who were surgically treated at University Hospital of Neiva, between the years 2005-2009. 41 people injured by APLM-UXM were chosen by checking records of pathology and medical histories. Among the findings it was found that most of those affected by APML-UXM are young military men, whose lower limbs are the most affected.

All of the injured people underwent surgical amputation of the affected limb, and more than halfof them began rehabilitation with the adaptation of a prosthesis. This public health problems hows the degradation of the armed conflict, with greatimpact on the rural population and deservesen forcing the treaty to banthese weapons. It is necessary to make emphasis on prevention and educational programs in risky areas, whilea negotiated solution to the internal armed conflict is sought.
\end{abstract}

Key words: Landmines, unexploded munitions, traumaticamputation, trauma management, lower limb injuries.

1 MD, Ph.D. en Salud Pública, Departamento de Medicina Social y Preventiva. Docente de Planta, Facultad de Salud, Universidad Surcolombiana. Correo electrónico: gastaiza@usco.edu.co

2 Médico. Organización Panamericana de la Salud.

Recibido: 23/09/2014 - Revisado: 28/11/2014 - Aceptado: 29/12/2014 


\section{Introducción}

Colombia vive desde hace aproximadamente cinco décadas un complejo Conflicto Armado Interno - CAI- con la intervención de varios actores armados ${ }^{[1-3]}$. De ahí, que la confrontación armada muestra una tendencia a la degradación progresiva con el uso de métodos de extrema crueldad por los diferentes actores ${ }^{[2]}$, convirtiendo los medios en fines y en pos de la primacía de intereses particulares y grupales sobre intereses generales de la población, configurándose en una violación al Derecho Internacional Humanitario - DIH_ ${ }^{[4]}$. Lo anterior se evidencia con el gran número de actos violentos acaecidos a diario en el territorio nacional, como son: el homicidio, el secuestro, las masacres, los desplazamientos masivos, las violaciones a la misión médica, y el uso de las minas antipersonal ${ }^{[5]}$; que generan consecuencias negativas cada vez más graves sobre la morbimortalidad, de la población civil, con secuelas en las condiciones de vida, y el sector salud en Colombia ${ }^{[6-8]}$.

E1 uso de minas antipersonal-MAP es un indicador importante del conflicto armado en Colombia; y se confirma a la vez que el país es catalogado como el único país en América Latina donde los actores armados siguen colocando dichos artefactos ${ }^{[9]}$ El pais ocupa en el mundo el tercer lugar después de Afganistán y Camboya ${ }^{[10,11]}$. La dificultad en su detección de estos artefactos, sus efectos fatales y graves daños físicos como: mutilaciones, amputaciones, pérdida de órganos vitales, y un complejo de secuelas psicológicas en las víctimas, los convierten en un arma altamente peligrosa que viola el DIH. Una mina puede ser letal hasta 50 años después se ser sembrada en el suelo; esto prolonga en el tiempo la acción de los grupos armados dilatando el conflicto $\mathrm{y}$ sus graves efectos ${ }^{[12,13]}$.

Según Monitor Landmine ${ }^{[14]}$, de 1999 a 2009, se identificaron 73576 eventos por MAP y MUSE, resultados que incluyen 119 paises o áreas; 51711 lesionados; $\mathrm{y}, 17867$ muertos. Geográficamente los eventos se distribuyen así: Asia y el Pacífico donde el 17,6\% de los países están afectados, y aportando el $45,7 \%$ de los eventos, sigue África con $66,7 \%$ de países afectados y aportando el $22,3 \%$, luego el medio oriente y el norte de África con una afectación del $94,4 \%$ de los paises, y correspondiéndole el $11,6 \%$ de los casos, luego, América con el $40 \%$ de paises afectados, y aportando el 9,8 de casos, por último están los países de la Commonwealth independientes los cuales la totalidad están afectados y aportan el 6,3 de casos, por último, Europa, con afectación del $54,8 \%$ de sus países y aportando el 4,3\% de casos. En ese periodo con más de mil eventos, los países más afectados fueron Afganistán, Camboya, Colombia, Irak e India. En América Colombia ocupa el primer lugar.

En el mundo en el 2009 hubo 3956 casos por MAP y MUSE, de la cuales 2855 fueron lesionadas y 1041 fueron muertos. E1 género más afectado fueron los hombres jóvenes con el 88\%.

En Colombia el comportamiento de las lesiones por MAP y MUSE, de 1990 a 2009 según el Programa Presidencial para la Acción Integral contra Minas ${ }^{[15]}$ fueron 4850 casos, con una proporción de $96,31 \%$ de eventos por MAP y el resto por MUSE. Antioquia fue el departamento más afectado, aportando un cuarto de los casos, seguido del Meta con el 10,14\%, el Caquetá con el $8,06 \%$, Norte de Santander con el $6.56 \%$ y Nariño con el $6,47 \%$. Los municipios más afectados están Tame, Vistahermosa, San Vicente del Caguán, Puerto Rico, Ituango y San Carlos.

De acuerdo a los años éste fenómeno se ha incrementado presentando en los años 2003 y 2004 a 2007 la mayor proporción de casos en este periodo, siendo en este último el mayor con el 11,98\% volviendo a decrecer. Las zonas rurales son las más afectadas aportando la casi totalidad de los casos $(98,04 \%)$. Los mayores de 18 años son los más afectados aportando el 90,13\% de los casos. Los miembros de las fuerza pública aportan el 62,08\% de los eventos. Más de dos tercios resultaron heridos 78,04\% y el resto muertos. E1 género más afectado es el masculino con el $94,42 \%$ de casos.

E1 departamento del Huila según el Programa Presidencial, aportó el $1.81 \%$ de los casos del periodo de 1990 a 2009 , con 88 casos. La casi totalidad de casos fueron eventos producidos por minas $(98,86 \%)$. Los municipios con más casos fueron Algeciras $29.55 \%$, Baraya y Colombia con $14,77 \%$, Neiva 10.23 , Isnos $7,95 \%$, Tello 5.68\%. El pico de casos de éste periodo en el departamento se dio entre el 2006 al 2009, siendo el 2008 el de mayor proporción de casos $18,8 \%$ después del cual descendió. El 96,59\% de los casos ocurrieron en elárea rural. Las personas mayores de 18 años fueron las más afectadas $89,25 \%$, y la fuerza pública fue la condición que aportó dos tercios de los afectados $(77,85 \%)$, los heridos fueron el $81,21 \%$ de los casos, el género masculino con el $95,97 \%$ de los $\operatorname{afectados}^{[16-18]}$.

De este fenómeno no existe conocimiento sobre las características de las personas atendidas que fueron lesionadas por MAPMUSE, como parte del impacto del conflicto armado interno CAI sobre la vida y la salud en esta región de Colombia. Por lo anterior, la investigación caracterizó las lesiones de las personas afectadas por MAP-MUSE que fueron atendidas quirúrgicamente en el Hospital Universitario de Neiva, 2005-2009.

\section{Materiales y métodos}

Se realizó un estudio cuantitativo, descriptivo, serie de casos, retrospectivo, que buscó explorar y caracterizar los lesionados por minas antipersonales MAP y municiones sin explotar MUSE atendidos quirúrgicamente en el Hospital Universitario de Neiva (HUN) entre los años 2005-2009. El Hospital Universitario Hernando Moncaleano Perdomo HUN es un sitio de atención de cuarto nivel de referencia de la region, donde se atiende casos de alta complejidad y es donde reposan los archivos de las personas atendidas por los eventos con MAP-MUSE.

Se tomaron los registros de patología y las historias clínicas de las personas afectadas por minas antipersonal. La muestra fue no representativa de los casos hallados en el servicio de patología y que tuvieran historia clínica, tomada con los siguientes criterios de inclusión: a) persona con lesión corporal por MAP-MUSE o cualquier artefacto explosivo, b) procedencia del departamento del Huila y vecinos c) Atención quirúrgica con amputación. Criterios de exclusion: Personas con amputaciones por entidades patológicas médicas, o traumáticas diferentes a las buscadas en el estudio. La muestra fueron 41 personas afectadas por MAP-MUSE para el estudio.

Durante el procedimiento de recolección inicialmente se revisaron los registros anatomopatológicos en el departamento de patología del Hospital para identificar las personas con lesiones quirúrgicas por artefactos explosivos, luego, se revisaron las historias clínicas de los casos, recolectando los datos en un formulario con las variables del estudio.

Los datos se tabularon y analizaron en el programa Epi-Info ${ }^{\circledR}$. Se hizo análisis estadístico descriptivo uni-variado y bivariado. Los investigadores guardaron confidencialidad y el anonimato de los datos de los registros e historias revisados, soportado por la 
R.F.S Revista Facultad de Salud

Julio - Diciembre de 2014;6(2): 20-25
Lesiones por minas antipersona y munición sin explotar, Hospital Universitario de Neiva, 2005-2009/Gilberto Mauricio Astaiza Arias et al.
Resolución 008430/1993 del Ministerio de Salud de Colombia, investigación aprobada por el comité de historias clínicas del Hospital.

\section{Resultados}

Se revisaron 41 reportes de patología y las historias clínicas de los lesionados con MAP-MUSE atendidos quirúrgicamente en el Hospital Universitario de Neiva entre el 1 de enero de 2005 y el 31 de diciembre del 2009

La edad de los afectados tiene una media de 25,4 años, con una desviación típica de 7,0 años, lo que significa que son personas jóvenes. La edad mínima es de 12 años y la máxima de 50 años. La mediana es de 24 años, y el $75 \%$ de las personas está por debajo de los 27 años. Por género, es de resaltar que solo hubo una mujer $(2,4$ $\%$ menor de 15 años afectada por MAP. La mayoría fueron hombres, un tercio de ellos, de 21 a 25 años de edad, seguidos del grupo de 16 a 20 años (Figura 1 ).

Dos tercios de las personas afectadas por las MAP-MUSE fueron militares y en menor proporción los otros afectados eran agricultores y estudiantes. Con respecto a los lugares de origen, en dos tercios de ellos fue desconocido porque eran militares, dado que los accidentes ocurrieron en diferentes lugares del departamento. El otro tercio de los afectados, igual al anterior, no presentaba este dato en la historia clínica

E1 2008 fue el año con mayor, frecuencia de estos eventos (27\%), seguido con el $23 \%$ en el 2006 , y el con el $19 \%$ en el 2009 . En más de la mitad de los lesionados no se conoce el municipio de origen donde ocurrieron las lesiones por MAP-MUSE, otros son Colombia, Planadas y Algeciras (Tabla 1)

Los lugares donde los lesionados recibieron la atención médica inicial fueron en el caso de los militares el dispensario militar con el $58 \%$ del total. La remisión y atención médica posterior se realizó en todos los lesionados en el Hospital Universitario de Neiva a donde llegaron remitidos.
Tabla 1. Características demográficas afectados por minas antipersona MAP y municiones sin explotar MUSE, Hospital Universitario Hernando Moncaleano Perdomo de Neiva 2005-2009

\begin{tabular}{lcc}
\hline Orupación & $\mathbf{n}$ & porcentaje \\
\hline Agricultor & 4 & 9,8 \\
Estudiante & 3 & 7,3 \\
Militar & 16 & 63,4 \\
Sin información & 8 & 19,5 \\
Total & 41 & 100 \\
\hline
\end{tabular}

Municipio de procedencia donde ocurrió el evento

\begin{tabular}{lcc}
\hline Colombia & 3 & 7,3 \\
Florencia & 1 & 2,4 \\
Guayabal & 1 & 2,4 \\
Planadas & 2 & 4,9 \\
Militar (origen desconocido) & 26 & 63,4 \\
Sin información & 8 & 19,5 \\
Total & 41 & 100
\end{tabular}

Sitio de atención inicial de afectados por MAP-MUSE

\begin{tabular}{lcc}
\hline Hospital de Algeciras & 4 & 9,8 \\
Puesto de salud de Balsillas & 1 & 2,4 \\
Hospital de Colombia & 5 & 12,2 \\
Establecimiento de sanidad militar & 17 & 41,5 \\
Hospital de Florencia & 1 & 2,4 \\
Puesto de salud Guayabal & 1 & 2,4 \\
Hospital de la Plata & 1 & 2,4 \\
Hospital de Planadas & 3 & 7,3 \\
Sin información & 8 & 19,5 \\
Total & 41 & 100
\end{tabular}

Fuente: elaboración de los autores.

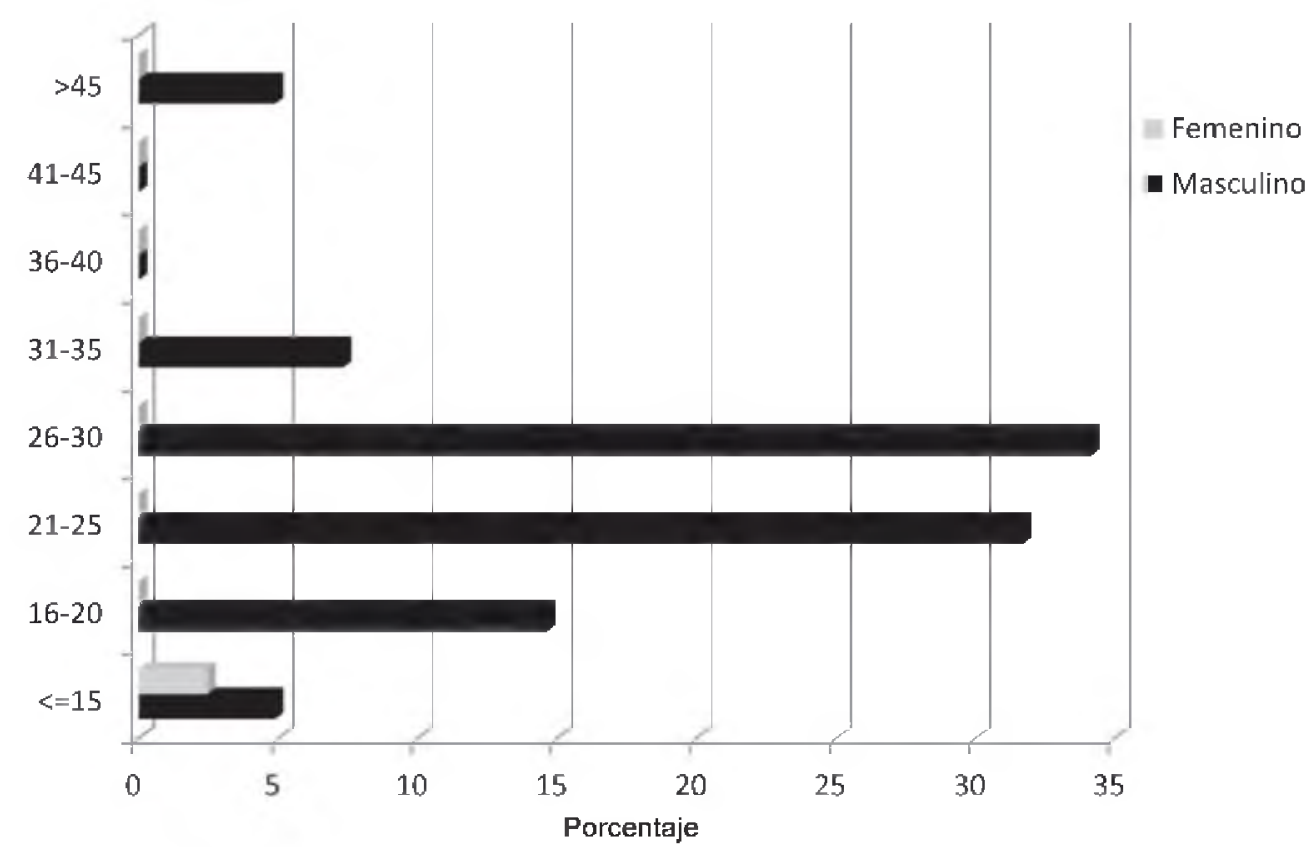

Figura 1. Distribución por edad y sexo afectados minas antipersona MAP y munición sin explotar MUSE, Hospital Universitario Hernando Moncaleano Perdomo de Neiva 2005-2009. 
Las actividades cotidianas que realizaban las personas afectadas al momento de explotar la MAP-MUSE eran: Patrullar en el caso de los militares con el 54\%, en los demás afectados está el trabajo agrícola y el desarrollo de actividades lúdicas, y un tercio aproximadamente carece de información (Tabla 2). Tres cuartas partes de las lesiones fueron causadas por minas antipersonal (MAP), el resto fueron por municiones sin explotar (MUSE). El sitio de localización de las MAP-MUSE, dos tercios son el suelo y del resto no hay dato. El mecanismo de activación del dispositivo en dos tercios de los lesionados fue mediante la presión con el pie cuando caminaban, seguido de la manipulación del dispositivo explosivo.

En los afectados la explosión del dispositivo produjo de una a tres lesiones. E1 90,2\% de las personas sufrieron sólo una lesión.

En cuanto a las extremidades afectadas, casi la mitad de las lesiones se produjo en el miembro inferior derecho, y en igual proporción en el miembro inferior izquierdo (Tabla 3).

Todos los lesionados recibieron manejo médico-quirúrgico con amputación de la extremidad lesionada en el Hospital Universitario. Los niveles de amputación efectuados con mayor frecuencia fueron a nivel del pie $40 \%$, y a nivel supracondíleo $30 \%$

Dos tercios de los lesionados amputados iniciaron el proceso de rehabilitación. A estos igualmente se les hizo la adaptación de una prótesis. Se desconoce la evolución que han tenido los lesionados en este proceso y como ha sido la incorporación a la vida cotidiana después del accidente.

Tabla 2. Características de la activación y del dispositivo de minas antipersona MAP y municiones sin explotar MUSE, Hospital Universitario Hernando Moncaleano Perdomo de Neiva 2005-2009

\begin{tabular}{lcc}
\hline Labor realizada activación MAP- MUSE & $\mathbf{n}$ & porcentaje \\
\hline Actividad agrícola & 3 & 7,3 \\
Caminar & 1 & 2,4 \\
Desactivación & 1 & 2,4 \\
Jugar & 2 & 4,9 \\
Patrullar & 22 & 53,7 \\
Sin información & 12 & 29,3 \\
Total & 41 & 100 \\
\hline
\end{tabular}

Tipo de artefacto explosivo

\begin{tabular}{lcc}
\hline Mina antipersonal MAP & 30 & 73,2 \\
Municiones sin explotar MUSE & 11 & 26,8 \\
Total & 41 & 100 \\
Localización aparato explosivo & & \\
Tierra & 27 & 65,9 \\
Otro sitio & 1 & 2,4 \\
Sin información & 13 & 31,7 \\
Total & 41 & 100 \\
\hline Forma de activación de MAP-MUSE & & \\
Manipulación & 4 & 9,8 \\
Presión & 26 & 63,4 \\
Sin información & 11 & 26,8 \\
\hline Total & 41 & 100 \\
\hline
\end{tabular}

Fuente: elaboración de los autores.
Tabla 3. Características de las lesiones por Minas antipersona MAP y municiones sin explotar MUSE, Hospital. Universitario Hernando Moncaleano Perdomo de Neiva 2005-2009.

\begin{tabular}{lcc} 
Número Miembros Lesionados & $\mathbf{n}$ & porcentaje \\
Uno & 37 & 90,2 \\
Dos & 3 & 7,3 \\
Tres & 1 & 2,4 \\
Total & 41 & 100 \\
\hline
\end{tabular}

Miembro Lesionado

\begin{tabular}{lcc} 
Inferior derecho & 18 & 43,9 \\
Inferior izquierdo & 18 & 43,9 \\
Superior derecho & 6 & 14,6 \\
Superior izquierdo & 4 & 9,8 \\
Total & 41 & 100 \\
\hline
\end{tabular}

Fuente: elaboración de los autores.

\section{Discusión}

Este estudio analiza la morbilidad y lesiones por las MAP-MUSE atendidas quirúrgicamente en el Hospital Universitario del Huila del 2005 al 2009

El impacto de este fenómeno a largo plazo se expresa en la discapacidad y repercusiones sociales en las personas afectadas tanto militares como civiles ${ }^{[1]}$. La anterior expresión es uno de los múltiples efectos que genera el conflicto armado interno en la salud de los colombianos ${ }^{[19]}$

En cuanto a la ocupación de la mayoría de personas afectadas por el evento se demuestra coherencia con lo encontrado en la literatura en donde se plantea que el despliegue militar y las situaciones traumáticas van a menudo de la mano, así mismo se dice que la guerra da lugar a importantes y persistentes trastornos psicológicos en la población civi[ ${ }^{[5,20]}$

En este orden de ideas es importante mencionar que "hay pruebas abundantes de la existencia de una prevalencia elevada de trastornos psiquiátricos entre las personas que tienen la mala fortuna de haber sufrido una guerra, como combatientes o como población civil atrapada en el seno de un conflicto de gran envergadu$\mathrm{ra}^{\mathrm{p}[21]}$ y aún más cuando son víctimas de las MAP-MUSE

La media de las lesiones es representativa y aunque en este estudio sólo se evaluó el grado de lesiones físicas adquiridas por las víctimas, se hace necesario mencionar que estas personas están expuestas a sufrir alteraciones en su salud mental tal como lo demuestran diferentes estudios como por ejemplo el realizado en SRI LANKA en donde se aplicó una encuesta epidemiológica sobre la población civil luego de la guerra, en la que se detectaron secuelas psicosociales en el 64\% de la población: Somatización (41\%), TEPT $(27 \%)$, trastorno de ansiedad (26\%), depresión mayor (25\%), alcoholismo y problemas relacionados con sustancias (15\%) y discapacidad funcional $(18 \%)^{[22]}$.

Teniendo en cuenta que el fenómeno de MAP-MUSE se presenta en estados que viven en guerra con otras naciones o incluso a nivel interno como en el caso de Colombia es importante destacar que "en muchos estudios se ha observado la existencia de una correlación directa entre la intensidad de la situación traumática y la 
gravedad de los problemas psicológicos. Cuanto mayor es la exposición a la situación traumática (tanto física como psicológica), más intensos son los síntomas" ${ }^{2[20]}$. Quizá lo alarmante de los efectos en la salud fisica y psicológica de los eventos de MAP-MUSE de la población tanto civil como militar es el hecho de que en estudios recientes se ha sugerido la transmisión intergeneracional de los efectos de los traumas psicologicos, lo que refuerza, adicionalmente, el impacto profundo y a largo plazo de la guerra ${ }^{[5]}$

De acuerdo a la totalidad de las lesiones, amputaciones y demás compromisos orgánicos, es necesario conocer los grados y niveles de dependencia de las victimas de MAP-MUSE en las actividades básicas de la vida diaria ya que conforme al grado de afectación física los niveles de dependencia se pueden clasificar en moderada, severa o gran dependencia, esta última cuando la persona necesita ayuda para realizar varias actividades básicas de la vida diaria (ABVD) varias veces al día, y por su pérdida total de autonomía física necesita el apoyo indispensable y continuo de otra persona para movilidad personal ${ }^{[23,24]}$. Además es indispensable categorizar la discapacidad que tiene la víctima teniendo en cuenta el modelo de discapacidad propuesto por la clasificación internacional del funcionamiento, la discapacidad y la salud ${ }^{[24]}$.

Es importante mencionar que sólo un porcentaje de los lesionados iniciaron el proceso de rehabilitación de los cuales se desconoce la evolución e incorporación a la vida cotidiana después de la lesión. En este sentido tal como lo señala Murthy, "ni la muerte es el único resultado de la guerra (la morbilidad psiquiátrica y la discapacidad están entre los abundantes resultados negativos de los conflictos armado), ni las víctimas son únicamente los militares profesionales. La población civil, a la que no se le ahorra ninguno de los horrores de la guerra, constituye el objetivo principal del terrorismo ${ }^{2,[20,25]}$.

La OMS calcula que en países (de bajos ingresos) en conflicto, el 10\% de las personas que viven experiencias traumáticas suelen tener graves problemas de salud mental (depresión y estrés postraumático) y otro $10 \%$, comportamientos que dificultarán su capacidad para vivir con normalidad, dado de que cuando el malestar mental es crónico, está asociado con una productividad disminuida, consecuencias desfavorables en cuanto a alimentación, educación, salud y procesos débiles de participación ${ }^{[26]}$.

Por otra parte, la investigación del conflicto armado y sus efectos en la salud de las victimas presenta algunas dificultades. Una de ellas es la poca disponibilidad de las fuentes de datos, situación que afectó en algún momento la viabilidad del proyecto. Otra es la localización de las personas afectadas para indagar sus circunstancias y vivencias por el evento, sobre todo en el caso de los militares, principales victimas de las MAP, dado que esa información es reservada por el Ejército Nacional.

También se evidenció sub-registro e inadecuada definición diagnóstica de los lesionados en el Hospital, dado que estos no aparecen definidos como eventos producto de MAP-MUSE, ante la inexistencia de una variable que los agrupe, por lo general, se registran como emergencias médico quirúrgicas generales o comunes.

En cuanto a los resultados, existe analogía con los resultados de otros estudios ${ }^{[27-29]}$ en lo referente a la distribución por sexo, edad, ocupación de los afectados, resaltando que la mayoria de las victimas procedian de áreas rurales y que son personas jóvenes del género masculino. Por lo general la amputación es la lesión más frecuente, afectando una o más extremidades ${ }^{[8,9]}$.

La presencia de las MAP - MUSE y sus víctimas, será un problema de salud pública que persistirá en el tiempo, en la medida de la permanencia del conflicto armado en Colombia. La morbi-mortali- dad por este fenómeno seguirá impactando gravemente los servicios de atención en salud, presionando a los servicios de urgencias, ya sobrecargados con la atención de las victimas de otras formas de violencia $^{[10]}$

La atención integral de ésta problemática exigirá del Estado la definición de varios enfoques en su accionar. Uno, creación de equipos multidisciplinarios para la intervención integral en el segundo y tercer nivel de prevención de los servicios de salud. Paralelamente deberá fortalecerse la prevención primaria, en especial con el cumplimiento de los convenios internacionales ratificados por el pais para la no proliferación de estas armas por parte del estado y los grupos armados, la aplicación intensiva de nuevos mecanismos y programas de desminado ${ }^{[22,26,30]}$, la implementación de campañas educativas para la identificación, y señalización de áreas de alta contaminación con MAP-MUSE, difusión de guias para que las comunidades conozcan los pasos a seguir cuando requieran de la atención inicial a los lesionados ${ }^{[31,32]}$. Lo anterior, de la mano con una intensa sensibilización hacia los actores armados, para la adopción de los protocolos de prohibición internacionales de las MAP y del respeto por el Derecho Internacional Humanitario de gran importancia en el desarrollo postconflicto ${ }^{[33-35]}$.

\section{Conclusiones}

En este estudio se evidencia como las MAP y MUSE producen serias lesiones fisicas debido a su alto poder destructor y como afecta en su mayoría a hombres jóvenes militares, con gran deterioro de la calidad de vida y su gran impacto socio-económico, debido a las grandes incapacidades que producen las amputaciones y lesiones que dejan en la anatomía.

E1 Hospital Universitario de Neiva es el centro de referencia de alta complejidad para la región Surcolombiana. Las víctimas de la violencia hacen compleja y difícil su labor asistencial en la medida que persisten amplias zonas rurales "contaminadas" por estas armas, 10 que hace necesario la implementación de medios adecuados de referencia y contrarreferencia para la oportuna atención de los heridos.

Se deben implementar sistemas que permitan la mejor disponibilidad de datos sobre estos problemas de salud pública. Mejorar el registro en los sitios donde son atendidos los lesionados por las MAP-MUSE, generando categorías más precisas para estas entidades tanto en los formularios, como en el reconocimiento de las mismas por los profesionales de la salud, que mediante su capacitación posibilitará en futuras investigaciones poder encontrar datos más precisos para profundizar en las características del fenómeno de estudio a nivel bio-psico-social, especialmente para identificar cuáles pueden ser los factores protectores y potencializadores en su rehabilitación y adaptación a su nueva situación.

Por otro lado, sería importante allanar caminos que permitan a investigadores acceder a instituciones como el Ejército Nacional altamente afectadas por este fenómeno, y permitir ampliar el conocimiento de este evento de gran impacto en la salud pública del país.

\section{Agradecimientos}

Agradecemos al Hospital Universitario Hernando Moncaleano Perdomo de Neiva por permitir la realización de este estudio, su acompañamiento y supervisión, y a la Vicerrectoria de Investigación y Proyección Social de la Universidad Surcolombiana. 


\section{Referencias}

1. Mantilla V, S. Economía y conflicto armado en Colombia: los efectos de la globalización en la transformación de la guerra. Latinoamérica, 2012; pp. 55-73.

2. Franco, S., Momento y contexto de la violencia en Colombia. Revista Cubana Salud Pública, 2003;29(1): 18-36.

3. Wallerstein, N., Violencia en Colombia: reflexiones de una profesora visitante. Revista Facultad Nacional Salud Pública, 2000;18(2):101-115.

4. Internacional, A., "iDéjennos en Paz!» La Población Civil, Víctima del Conflicto Armado Interno de Colombia. 2008, Madrid España: Amnistía Internacional (EDAl).

5. Levav, I., El terrrorismo y sus efectos sobre la salud mental. World Psychiatry, 2006;4(1).

6. Pozzo, J. Las minas y el napalm. Dos problemas para ocuparse. Cuadernos de Marte, 2013

7. Castaño, B. Violencia sociopolítica en Colombia. Repercusión en la salud mental de las víctimas. 1994, Bogotá D.C.: Corporación Avre.

8. Moreno, C. Bermudez, If. Latorre and Debedout,r. Elsevier, potential of a hand transplantation program in Colombia. 43, 3529-3532 (2011).

9. Programa Presidencial para la Acción Integral contra Minas, Victimas por Minas Antipersonal (M.A.P.) y Municiones $\sin$ Explotar (MUSE) en Colombia 1990- 75 de julio 2008. 2008.

10. Rappert, Brian. Moyes, Richard. Lang, Lain. The case for addressing explosive weapons: conflicto, violence and Health. Science Direct, social science and medicine 2012;75:2047-2054.

11. International Campaign to Ban Landmines, Landmine Monitor 2010. Canadá: Mines Action Canada.

12. O.N.U., Minas Terrestres. 2007.

13. Gutiérrez Bolívar, J.A., Diseño Conceptual del Sistema Portante para un Sensor GPR en un Dispositivo para Detección de Minas Antipersona en Antioquia-Colombia in Escuela de Mecatrónica-Facultad de Minas. 2011, Universidad Nacional de Colombia: Medellín. p. 74.

14. Landmine Monitor, Landmine Monitor Report 2009. Toward a Mine-Free World. 2009, Canada: Landmine Monitor Editorial Board.

15. Programa Presidencial para la Acción Integral contra Minas, Estadísticas 1990-2014 víctimas minas antipersonal. 2014

16. Zuleta, N., 2005.

17. Landmine and Cluster Munition Monitor, Víctimas en el 2009. 2009: http://www.the-monitor.org/custom/ index.php/region_profiles/translation/30

18. Programa Presidencial para la Acción Integral contra Minas. Sistema INSMA. Situación Nacional Víctimas de Minas Antipersonal en Colombia. 2012.
19. COLOMBIA. Política de prohibición de minas antipersonal. Publicado por Campaña Colombiana contra minas. Documento del capítulo del Monitor de Minas terrestres 2014.

20. Murthy, R. and R. Lakshminaryana, Consecuencias de la guerra sobre la salud mental: una breve revisión de los resultados de las investigaciones. World Psychiatry, $2006 ; 4(1)$.

21. Galea, S. and K. Wortman, La salud de la población como argumento frente a la guerra. World Psychiatry, 2006;4(1).

22. The Halo Trust. SRI LANKA 2014.

23. Osorio, L. Módulo de Amputados 2010.

24. Cortés, Reyes E. Riveros L, Gustavo A y Pineda-Ortiz. Clasificación internacional del funcionamiento, la discapacidad y certificación de discapacidad en Colombia. Revista de salud pública 2013;15(1):129-137

25. Programa Presidencial para la Acción Integral contra Minas, Convención sobre la Prohibición del Empleo, Almacenamiento, Producción y Transferencia de Minas Antipersonal y sobre su Destrucción. 2012: http:// www.accioncontraminas.gov.co/Documents/Desminado/ Articulo_7/Informe\%20de\%20Articulo\%207\% 202012.pdf.

26. OMS-OPS, Acción concertadas minas. 1998.

27. Al-Worikat, A., Landmine amputees referred to the Royal Medical Sevices-Jordan. Prosthetics and Orthotics International, $2001 ; 25: 108-112$.

28. Hernández, G., Minas antipersonales (M.A.P.) en Colombia, costo físico y emocional. Umbral Científico, $2003 ; 2$.

29. Suárez, F., et al. Flora Bacteriana en heridas de guerra. Experiencia de dos años en el Hospital Militar Central de Bogotá. Revista Med 2008;16(1):127-133.

30. Villamil, E., J. Gutiérrez, and H. Morales, Trauma vascular de guerra en Colombia: análisis de 13 años en el Hospital Militar Central, in Universidad Militar Nueva Granada-Hospital Militar Central de Bogotá. 2013, Universidad Militar Nueva Granada: Bogotá D.C. p. 56.

31. Programa Presidencial para la Acción Integral contra Minas Antipersonal, Afiche Ruta de Atención Integral a Víctimas de Minas Antipersonal (MAP) y Municiones sin Explotar (MUSE). 2008.

32. Restrepo, A. and J. López, Perfil clínico y microbiológico de las lesiones por minas antipersonal en el Hospital Pablo Tobón Uribe, Medellín, 2003-2005. Biomédica, 2010;30(3).

33. Barberán, M. Eliminemos las minas. Campaña por la prohibición total de la minas.

34. Naciones Unidas, Convención sobre la Prohibición del Empleo, Almacenamiento, Producción y Transferencia de Minas Antipersonal y sobre su Destrucción. 1997.

35. Presidencia de la República de Colombia, Decreto Número 2150 de 2007. 2007. 